\title{
Research on Distributed Jet Blowing Wing Based on the Principle of Fan-Wing Vortex-Induced Lift and Thrust
}

\author{
Du Siliang $\mathbb{D}^{1,2}$ Zhao Qijun $\mathbb{D}^{1}{ }^{1}$ and Wang Bo $\mathbb{D}^{1}$ \\ ${ }^{1}$ National Key Laboratory of Rotorcraft Aeromechanics, Nanjing University of Aeronautics and Astronautics, Nanjing 210016, China \\ ${ }^{2}$ Faculty of Mechanical \& Material Engineering, Huaiyin Institute of Technology, Huaian 223003, China
}

Correspondence should be addressed to Zhao Qijun; zhaoqijun@nuaa.edu.cn and Wang Bo; wangbo@nuaa.edu.cn

Received 24 December 2018; Revised 29 March 2019; Accepted 7 April 2019; Published 9 July 2019

Academic Editor: Kenneth M. Sobel

Copyright (c) 2019 Du Siliang et al. This is an open access article distributed under the Creative Commons Attribution License, which permits unrestricted use, distribution, and reproduction in any medium, provided the original work is properly cited.

\begin{abstract}
Based on the numerical calculation and analysis of the principle of the lift and thrust of the Fan-wing. A new scheme for the wing of Fan-wing aircraft-distributed jet blowing wing was presented. Firstly, the mechanism of the formation process of the vortex-induced lift and thrust force of the two kinds of wings was analyzed. Then, the numerical calculation method and validation example were verified. It was proved that the distributed jet blowing wing had the same vortex-induced lift and thrust mode as that of the Fan-wing by comparing the relative static pressure distribution curve, velocity contours, and pressure contours. Finally, the blow-up speed of a jet blowing wing was defined and the relationship between the lift and thrust of two wings with the flow speed and angle of attack was compared. The result indicated that the lift and thrust of the distributed jet blowing wing was similar to those of the Fan-wing under normal flight conditions. Therefore, it was proved that the Fan-wing can be replaced by the distributed jet blowing wing. Furthermore, distributed jet blowing wing technology has the potential value for application in an ultrashort take-off and landing concept aircraft.
\end{abstract}

\section{Introduction}

Various innovative approaches to achieve distributed lift and thrust have been proposed, i.e., blended wing designs with arrays of distributed engines [1]. Cyclogyros have been proposed and developed since the beginning of the 20 th century [2-4]. Some recent examples at various research centers show the potential of such systems [5]. Cyclogyros exhibit complex mechanics based on cams and control rods and require extra bearings to achieve the cyclic pitch of the airfoils. In comparison to cyclogyros, the Fan-wing [6] has a simpler and lower-cost construction, since the blade pitch angle is constant and thus mechanically fixed.

Fan-wing concept with distributed propulsion is described as a simple, stable, and very efficient high-lift aircraft wing. Compared with the common aircraft with a fixed wing, the relative thickness of its wing is greater and there is a crossflow fan with infinitely variable speed powered by the engine at the leading edge of each wing. The cross-flow fan pulls the air in from the front and accelerates the air over the trailing edge of the wing. Therefore, the Fan-wing accelerates a large volume of air and produces lift and thrust simultaneously. This kind of distributed lift and thrust of the Fan-wing has higher efficiency than that gained by improving the by-pass ratio of the gas turbine engine currently. The flow field around the wing section of a Fan-wing aircraft under various flow conditions has been analyzed by Bayindir and Guillermo [7]. The high lift characteristics of the Fan-wing were further proved. The advantages of a Fan-wing aircraft compared to a conventional aircraft are short take-off and landing (STOL) at the low forward speed, no stall, and high power load. Recently, the investigations on the Fan-wing technology integrated into airfoils showed the high lift potential of the embedded propulsion system and moved the research from experimentation to prototyping [8]. Several experimental programs have been carried out to demonstrate 
the Fan-wing concept, including the work of Peebles [9] at the University of Rome and the work of Foreshaw [10] and Kogler [11] at the Imperial College.

The new two-year research and development SOAR project is aimed at optimising the originally patented FanWing rotor and wing shape and explore the feasibility of a full-size cargo-lifting FanWing from 2013. The SOAR (diStributed Open-rotor AiRcraft) open-fan wing technology at the focus of this project is a new concept that distributes the thrust and powered lift over the entire span of the wing resulting in a model-proven lift efficiency of helicopters as well as truly quiet U-STOL (ultrashort take-off and landing) performance and safe autorotation landing. Up to now, the latest progress of the project has not been disclosed.

Our project team has been exploring and researching the Fan-wing since 2011 [12-15] and has achieved a lot, but to the manned or bigger Fan-wing aircraft, the diameter of a cross-flow fan should get larger with the increase of the aircraft's maximum takeoff weight. Furthermore, the crossflow fan was installed on the upper surface of the wing and occupied nearly half of the chord length of the wing so that objects like sand and birds will damage the fan blade and the rotating structure without necessary protective measures in case. In addition, the dynamic balance of cross-flow fan is more difficult than that of a helicopter rotor or aircraft propeller. These shortcomings limit the further development and application of this low-speed and large-load aircraft.

In this study, a kind of a distributed jet blowing wing (DJBW) was proposed, which used the active flow control technique to produce vortex-induced lift and thrust based on the principle of the Fan-wing. This concept maintains the advantage of the simple structure and no moving parts for the airflow acceleration. The compressed pressure air with high energy for airflow acceleration can be bled from a core engine or a compressor. So this kind of propulsion wing unit may be useful in the field of new concept vehicles.

\section{Fan-Wing Principle Exposition}

Figure 1(a) shows the flight rendering map of the Fan-wing aircraft. Figure 1(b) shows the sketch map of the lift and thrust force of the Fan-wing section. Figure 1(c) and Figure 1(d) show the velocity contour and the pressure contour of the Fan-wing based on the numerical calculation, respectively. It can be clearly seen that the airflow velocity at the installation of a cross-flow fan is $0 \mathrm{~m} / \mathrm{s}$. Vortex circulation phenomenon was formed in the installation area of the crossflow fan. Figure 1(e) shows the relative static pressure distribution curve on both the upper and the lower surfaces of the Fan-wing. Figures 1(c)-1(e) were derived from the numerical calculation method in the third part of this paper. Define $X=0 \mathrm{~mm}$ as the rotation center of the cross-flow fan. We can notice that the lift generated in the installation area of the cross-flow fan accounts for about $70 \%$ of the whole airfoil, which indicated that the high lift of the Fan-wing was caused by a low-pressure vortex. Vortex generation was caused by a cross-flow fan changing the direction of incoming flow and accelerating incoming flow. The continuous rotational motion of the cross-flow fan maintained the existence of vortices. It was different from the vortex ring induced by the trailing edge of an ordinary wing. It was an active vortex.

\section{Distributed Jet Blowing Wing Concept}

In the first part of this paper, the shortcomings of the Fan-wing in the future application have been introduced. Therefore, the flow control technology was considered to replace the role of the cross-flow fan in accelerating and changing the flow direction. High lift devices and flow control methods are aimed at significantly increasing the maximum lift of the wing without increasing its size. The flow control methods described in this thesis were based on the boundary layer principles first described by Prandtl in 1904 [16]. The vortex generators, flaps, and slats were called passive flow control because no external source of energy is supplied. In the passive flow control method, the energy is transferred from the main flow to the boundary layer. Conversely, the active flow control method requires an external source of energy. For the active flow control, the energy was transferred from this external source of energy (pump, aircraft compressor, plasma discharge, etc.) to the fluid. Schlichting and Gersten [17] presents a comprehensive overview of the boundary layer and flow control methods. Smith extended Schlichting and Gersten's work to include high lift aerodynamics, a study that encompasses the airfoil design, wing design, boundary layer control, and flow control methods [18]. A more recent overview from the DLR is available in [19].

Combined with the Fan-wing and active flow control technology, the DJBW was proposed for lift and thrust enhancement. The specific methods are as follows: In order to facilitate comparative analysis, remove the cross-flow fan from the Fan-wing. Two jet blowing ports were set at the apex of the airfoil. A jet blowing port was at the bottom of the airfoil arc groove. The blowing direction of blowing port 1 was blown backward along the trailing edge of the airfoil. The blowing direction of blowing port 2 was blown along the bottom of the airfoil arc groove. The blowing direction of blowing port 3 was blown from the bottom of the airfoil to the leading edge of the airfoil along the arc groove. Figure 2 illustrates the origin of the DJBW concept from the Fan-wing.

At present, the lift enhancement technology using flow control technology is external blown flap flow control technology developed in mid-50s by NASA, which augments the lift coefficient by directing the engine exhaust jets below the wings to flow through the highly deflected multielement flap systems. Therefore, the distributed jet blowing method proposed in this paper is feasible. Figure 3 shows a three-dimensional design of a new concept aircraft with the DJBW.

This study will start from the mechanism of the formation of the low-pressure vortex of the Fan-wing and simulate the acceleration effect on the flow from the cross-flow fan by arranging blowing ports in the proper position of the airfoil to study whether it will get similar aerodynamic characteristics to the Fan-wing's in this way. Firstly, a numerical model 


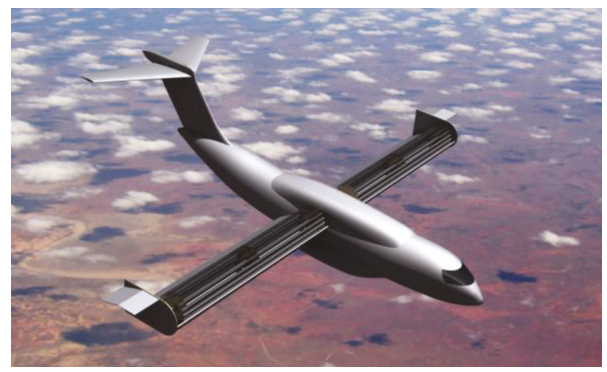

(a) Fan-wing aircraft

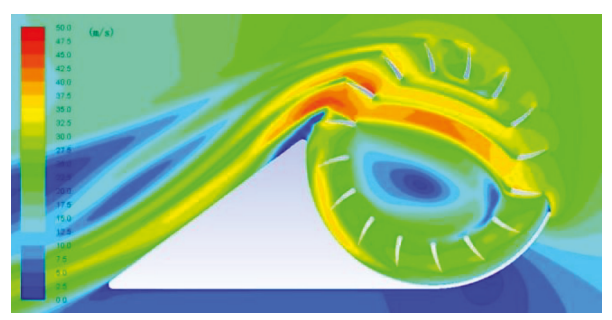

(c) Velocity contour of the Fan-wing airfoil

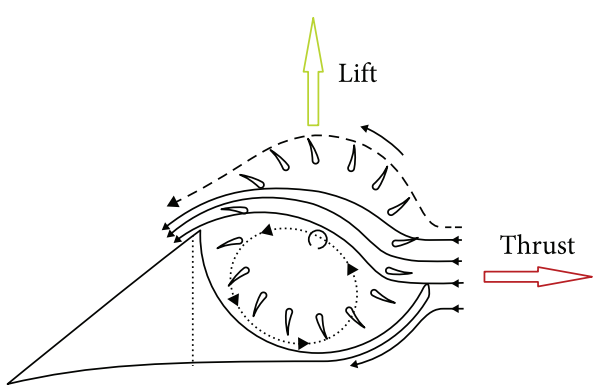

(b) Sketch map of Fan-wing lift and thrust force

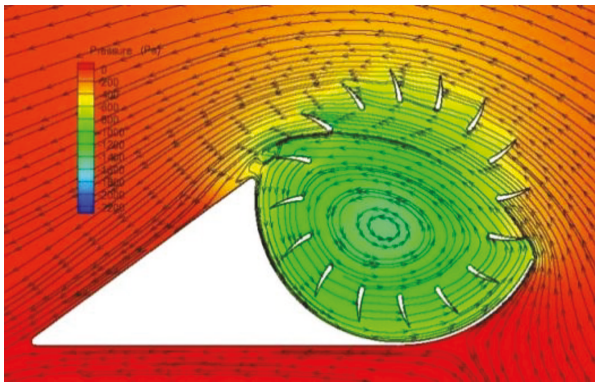

(d) Pressure contour and velocity flow chart of the Fan-wing airfoil

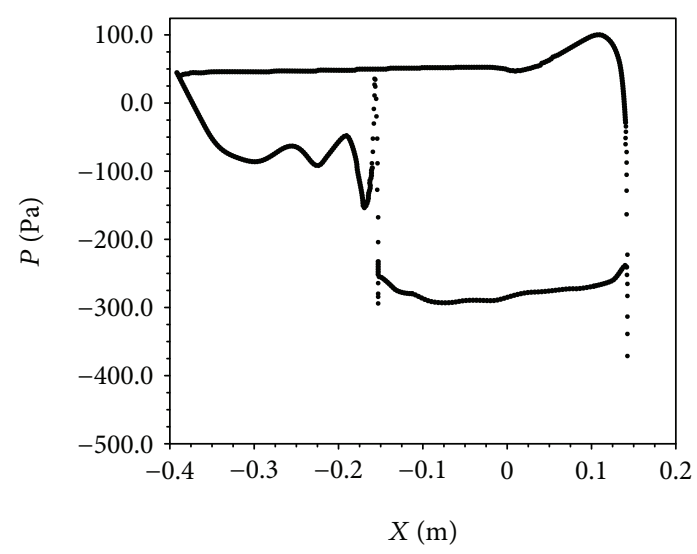

(e) Static pressure distribution curve of the Fan-wing airfoil

FIgURE 1: Introduction of the Fan-wing principle.

of the Fan-wing was established. Then, the blowing wing airfoil was built based on the thick airfoil of the Fanwing. The reliability of numerical calculation was verified by experiments. The acceleration data of the cross-flow velocity on airflow in the Fan-wing were obtained by the PIV (Particle Image Velocimeter) test, which was used to define the blowing speed of each blowing port. Finally, the aerodynamic characteristics of the two airfoils were preliminarily analyzed and compared, and the preliminary results were obtained.

\section{Numerical Method and Validation}

4.1. Model Establishment. Figure 4(a) shows the model of the Fan-wing used in the numerical calculation of this paper, whose length of the wing is $500 \mathrm{~mm}$. Figure 5 shows the basic geometric size of the wing cross-section. Some geometric parameters which have effects on airfoil are shown in
Table 1. The shape of the cross-flow fan blade was also one of the important parameters affecting the aerodynamic characteristics of the Fan-wing. Therefore, the definitions of blade parameters are shown in Table 2 and Figure 5(b). The physical model of the Fan-wing in the wind tunnel test is shown in Figure 4(b). The cross-flow fan was made of carbon fibers. The airfoil was made of glass fiber.

Figure 6(a) shows a three-dimensional model of the DJBW. Figure 6(b) shows a cross-sectional view of the DJBW. The three blowing ports all have air outlets with rectangular cross-sections. The height and the length of the ports were defined as $5 \mathrm{~mm}$ and $500 \mathrm{~mm}$, respectively. The rectangular blower was designed with reference to the inner blown flaps of an AG600 amphibious aircraft made by the Aviation Industry Corporation of China [20].

4.2. Calculation Method. The numerical simulations were used the commercial available general CFD code FLUENT 


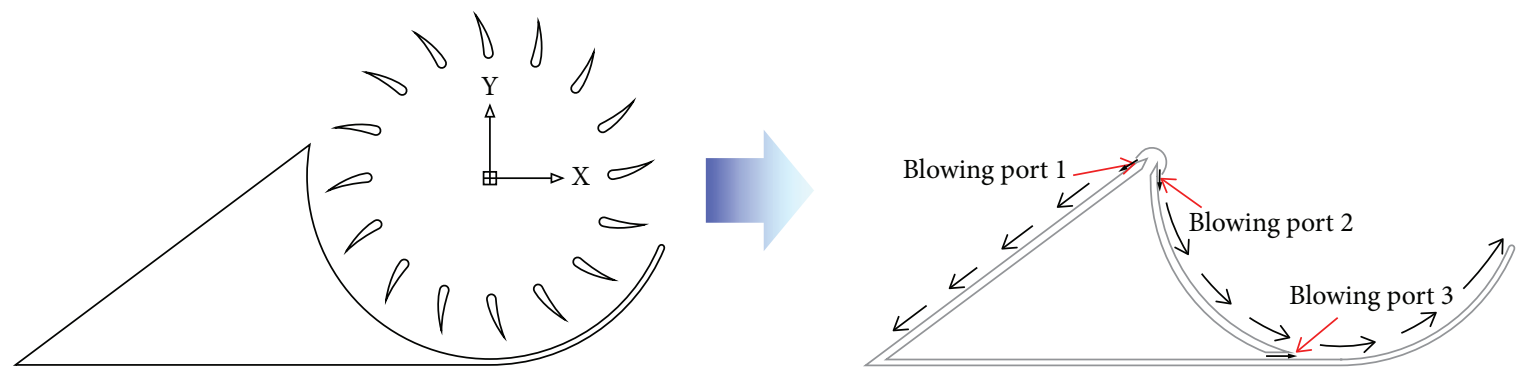

FIGURE 2: Evolution of the Fan-wing airfoil to evolution of the DJBW airfoil.

14.5 by Fluent Inc. Grid division used the ANSYS ICEM software. For this CFD analysis, the free stream velocity and angle of attack were constant for all rotation velocities. The renormalization group model was used for turbulence. The pressure-velocity coupling was calculated using the SIMPLEC algorithm. Second-order upwind discretization was considered for the convection terms. The rotating and stationary domains connected each other with a fluid-fluid interface used by the Fan-wing, where the flow continuity was satisfied. To simulate the fan rotation, the area surrounding the blades was designed as a sliding mesh region. All residual targets were set to less than $1 e-4$. If no signs of numerical instabilities to occurred after 1500 iterations, the solution was considered to have reached convergence. The boundary condition and the domain definition of the Fanwing model are shown in Figure 7 (a), in which $V$ and $P$ represent the velocity-inlet and pressure-outlet boundary conditions, respectively. The computational region of the flow field is shown in Figure $7(\mathrm{~b})$. The length and width of the computational domain are $14 \mathrm{~m}$ and $8 \mathrm{~m}$, respectively. The definition of the blowing airfoil boundary was the same. Figures $7(\mathrm{c})$ and $7(\mathrm{~d})$ show the mesh of the Fan-wing and DJBW generated by the ANSYS ICEM CFD software, respectively.

4.3. Example Verification. To verify the effectiveness of the numerical method used in this paper, with the help of a low-speed open return-flow tunnel provided by the National Key Laboratory of Science and Technology Helicopter Rotorcraft Aeromechanics in Nanjing University of Aeronautics and Astronautics, we measured the lift and the thrust of the Fan-wing. The experimental model is shown in Figure 8(b). We compared the numerical results with the experimental results of the lift and the thrust of the Fan-wing when the inflow velocity, angle of attack, and the rotation speed of the cross-flow fan were $12 \mathrm{~m} / \mathrm{s}, 10^{\circ}$, and $750 \sim 2000 \mathrm{r} / \mathrm{min}$, respectively. Figures 9 (a) and 9(b) show the test results of the lift and the thrust of the Fan-wing at the different rotation speeds of the cross-flow fan. As we can see from the figures, with the increase of cross-flow fan rotation speed, the lift and the thrust gradually increased as well and the calculation results coincide with the trend of test results well. Therefore, the numerical method mentioned above can be used in the calculation and analysis of the wing aerodynamic characteristics.

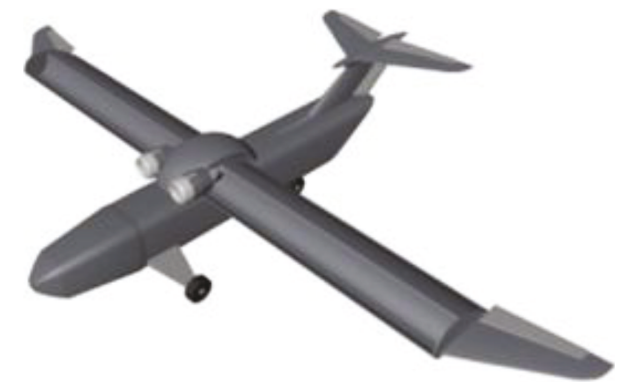

FIGURE 3: A new concept aircraft with a DJBW.

In order to obtain the acceleration speed of the cross-flow fan at different rotational speeds and set the initial blowing speed of the ports, PIV velocity measurement experiment was designed and compared with the numerical simulation results. Because of the shielding of the cross-flow fan blade, the airflow velocity in the cross-flow fan could not be measured, so the airflow velocity in the rear edge of the airfoil was only measured in this experiment. Figure 10(b) shows the velocity measurement area of the velocity measurement test. Figure 11 verifies that the calculation of airflow velocity in the numerical algorithm is credible.

\section{Calculation Results and Analysis}

5.1. The Numerical Simulation Calculation of the Blowing Wing. Setting the inflow speed as $12 \mathrm{~m} / \mathrm{s}$ and the angle of attack as $0^{\circ}$, we calculated the maximum airflow velocity on both the upper and the lower surfaces of the Fan-wing ranges from $30 \mathrm{~m} / \mathrm{s}$ to $50 \mathrm{~m} / \mathrm{s}$ as the rotation rate of cross-flow fan ranges from 1000 to $2000 \mathrm{rpm}$. Thus, we set the exit airflow velocity of the blowing wing's three blowing ports as $30-50 \mathrm{~m} / \mathrm{s}$. Initial assumption was that the blowing velocities of the three ports were the same. When the jet blowing ports velocity was $50 \mathrm{~m} / \mathrm{s}$, the velocity and pressure contours were calculated (Figures 12(b) and 12(d)), compared with the velocity and pressure contours of the Fan-wing (the rotation speed of cross-flow fan was $2000 \mathrm{rpm}$ ) in Figures 12(a) and 12(c). We can find that the DJBW produced elliptical low-pressure vortex as well and the streamline of airflow was similar to that of the Fanwing. In addition, the shape of low-pressure vortices was better than that produced by the cross-flow fan in the Fan- 


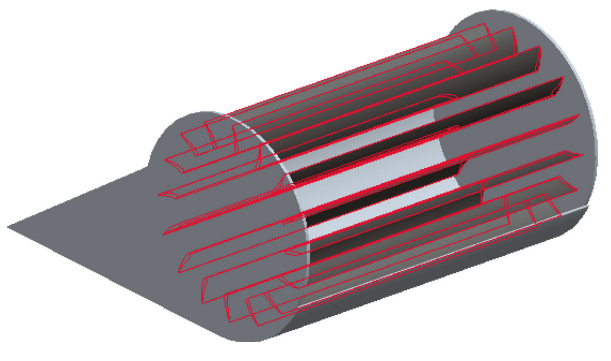

(a) CATIA model of a Fan-wing

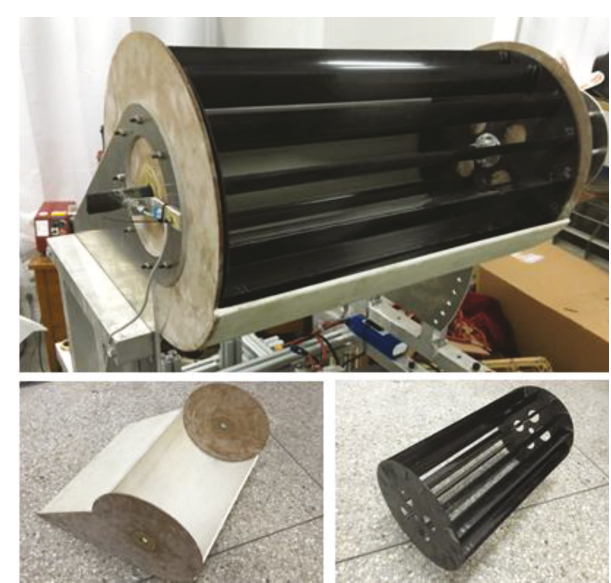

(b) Fan-wing test object

Figure 4: Fan-wing.

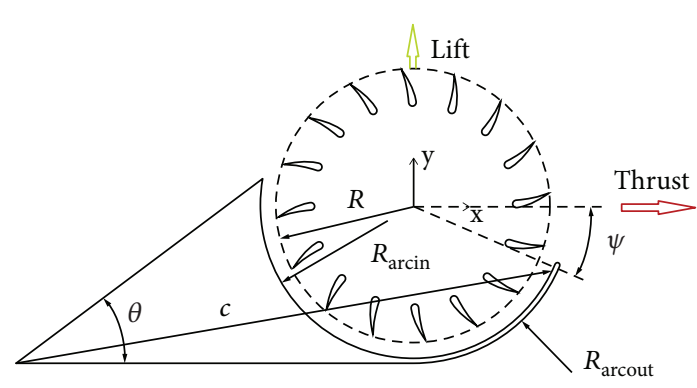

(a) Geometry of a Fan-wing airfoil

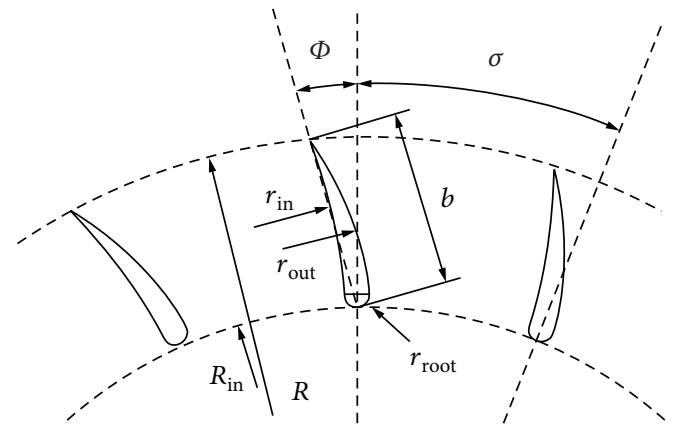

(b) Geometry of a cross-flow fan blade

FIgURE 5: Fan-wing section dimension parameter.

TABLE 1: Geometry of Fan-wing airfoil parameters.

\begin{tabular}{lc}
\hline Parameters & Value \\
\hline Radius of cross-flow fan $R(\mathrm{~mm})$ & 150 \\
Inner radius of semicircular cavity $R_{\text {arcin }}(\mathrm{mm})$ & 155 \\
Outer radius of semicircular cavity $R_{\text {arcout }}(\mathrm{mm})$ & 160 \\
Chord $c(\mathrm{~mm})$ & 561 \\
Trailing angle $\theta\left(^{\circ}\right)$ & 36.5 \\
Leading edge opening angle $\psi\left({ }^{\circ}\right)$ & 24 \\
\hline
\end{tabular}

TABLE 2: Geometry of cross-flow fan blade parameters.

\begin{tabular}{lc}
\hline Parameters & Value \\
\hline Blade width $b(\mathrm{~mm})$ & 36 \\
Outer radius of cross-flow fan $R(\mathrm{~mm})$ & 150 \\
Inner radius of cross-flow fan $R_{\text {in }}(\mathrm{mm})$ & 98 \\
Blade outer arc radius $r_{\text {out }}(\mathrm{mm})$ & 96 \\
Blade inner arc radius $r_{\text {in }}(\mathrm{mm})$ & 68 \\
Blade root arc radius $r_{\text {root }}(\mathrm{mm})$ & 3 \\
Blade installation angle $\phi(")$ & 18 \\
Contiguous blade angle $\sigma(")$ & 22.5 \\
\hline
\end{tabular}

wing. Figures 12(f) and 12(e) show the pressure contour and the velocity flow chart of the blowing wing as its three blowing ports' exit airflow velocity is $0 \mathrm{~m} / \mathrm{s}$ and of the Fan-wing as its rotation speed of the cross-flow fan is $0 \mathrm{~m} / \mathrm{s}$, respectively, from which we can find that the DJBW still can produce a low-pressure vortex by inflow in its arc groove even without blowing in its ports, while the Fan-wing produced turbulence inside the arc groove due to the obstruction of structures like the blade of the cross-flow fan, etc. Thus, the DJBW has a far better gliding performance than the Fan-wing. Figure 12(h) shows the relative static pressure distribution curve of the DJBW on both the upper and the lower surfaces, and compared with Figure $12(\mathrm{~g})$, it is also similar to that of the Fan-wing. And the pressure jump zone of the DJBW located at the top position of the arc groove (when $X=-0.15 \mathrm{~m}$ ) was smaller than that of the Fan-wing. Therefore, the scheme proposed in this paper, utilizing the generating mechanism of the Fan-wing lift and thrust, realizes the formation of the low-pressure vortex similar to the Fan-wing by changing the cross-flow fan which was used to accelerate the airflow into the blowing way to work.

5.2. Comparison of Aerodynamic Characteristics. Figure 13 presents the relation curve showing how the lift varies with 


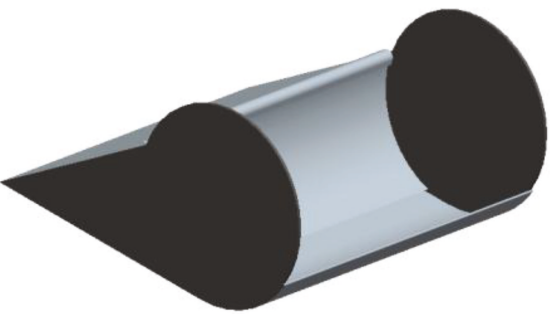

(a) Blowing wing airfoil

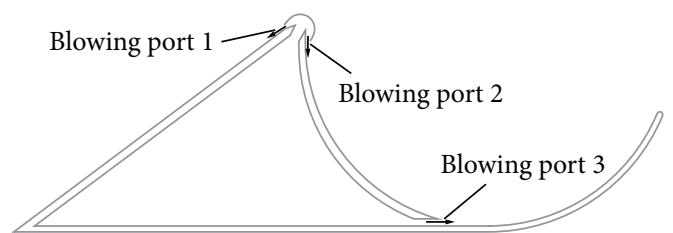

(b) Blowing airfoil section

FIgURE 6: Fan-wing section dimension parameter.

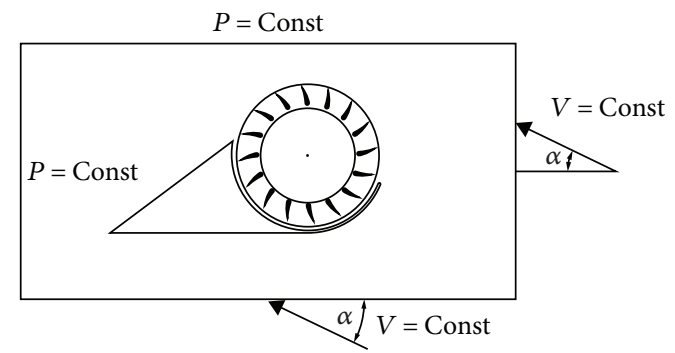

(a) Computational domain and boundary conditions

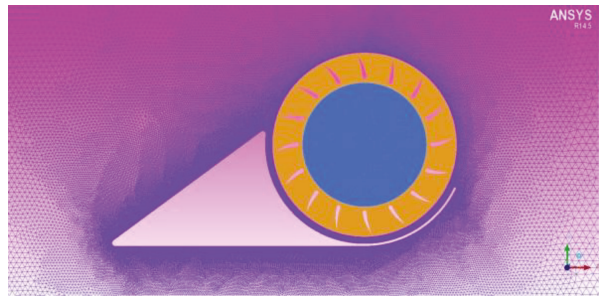

(c) Mesh of the Fan-wing

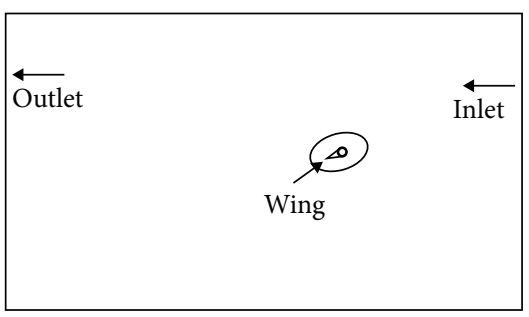

(b) Computational region of the flow field

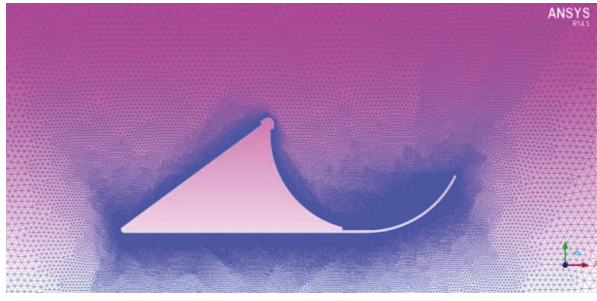

(d) Mesh of the DJBW

Figure 7: Mesh of two kinds of wings.

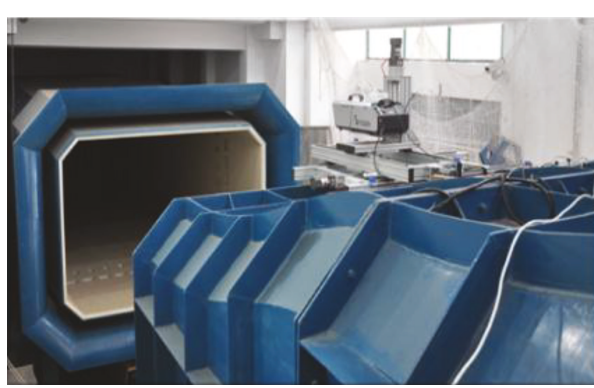

(a) Wind tunnel

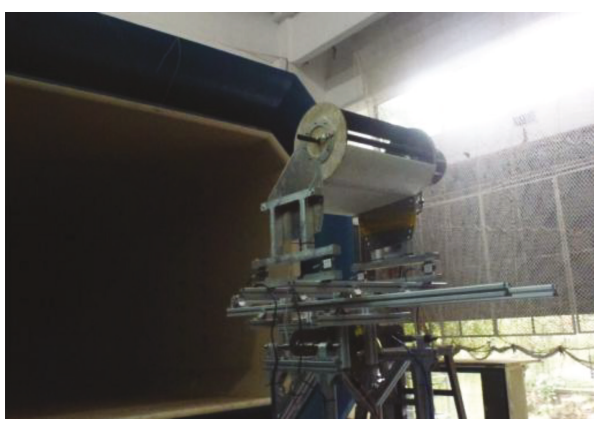

(b) Test bench

Figure 8: Experiment of the Fan-wing.

the changing rotation rate of the cross-flow fan as well as the changing blowing speed in blowing ports when the inflow velocity was $12 \mathrm{~m} / \mathrm{s}$ and the angle of attack was $10^{\circ}$ for both the Fan-wing and the DJBW. We can find that the lift produced by the two kinds of wings grows with the increase of the rotation speed and blowing speed. The increase rate of the DJBW slows down gradually while that of the Fan-wing was small in low rotation speed and on the contrary in high rotation rate, which was caused by unevenly accelerated airflow of the cross-flow fan.

Figure 14 presents the relation curve showing how the thrust varies with the changing rotation rate of the cross- 


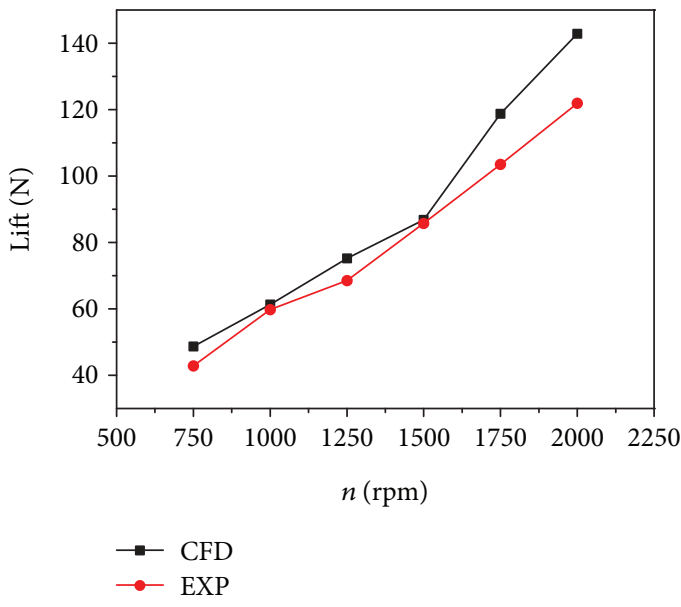

(a) Lift of comparison

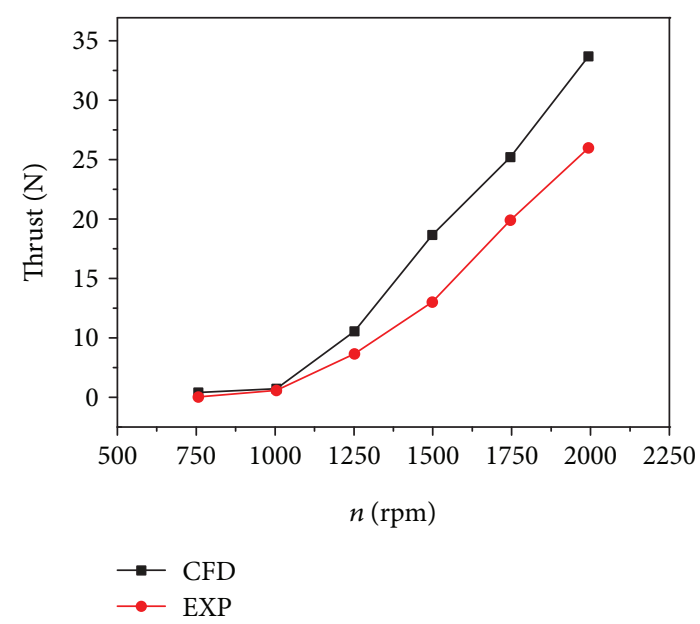

(b) Thrust of comparison

Figure 9: Comparison chart of the Fan-wing.

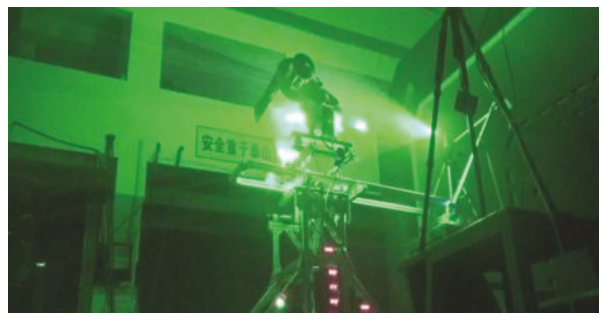

(a)

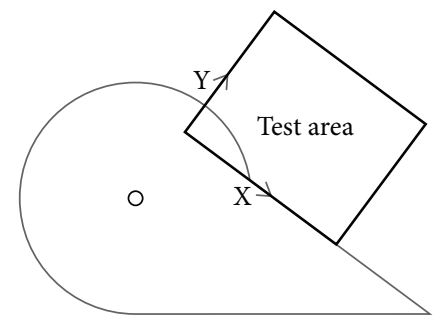

(b)

FIGURE 10: PIV velocity measurement experiment.

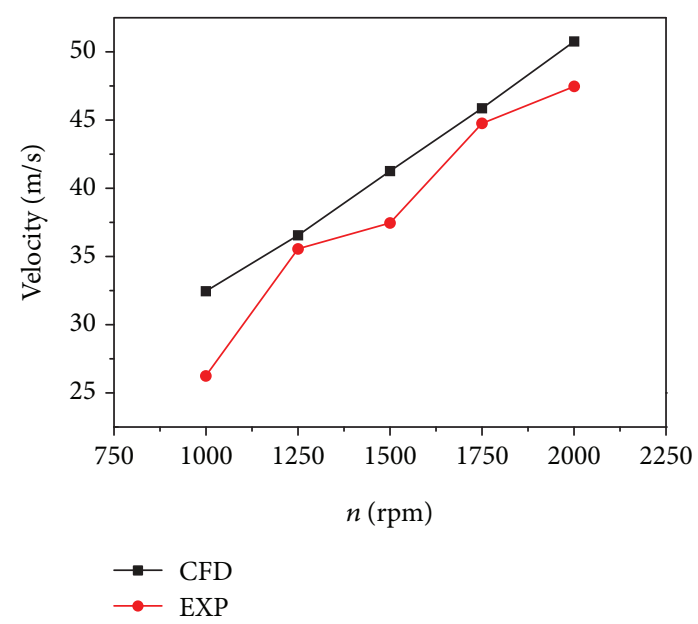

FIGURE 11: Comparison chart of the test and numerical value of velocity.

flow fan as well as the changing blowing speed in blowing ports when the inflow speed is $12 \mathrm{~m} / \mathrm{s}$ and the angle of attack is $10^{\circ}$ for both the Fan-wing and the blowing wing. We can find that the thrust produced by the two kinds of wings grows with the increase of the rotation speed and blowing speed, with which the increase rate of the DJBW was more consistent while that of the Fan-wing was fluctuant though in general accord.

Figure 15 presents the relation curve showing how the lift varies with the changing angle of attack $(\alpha)$ when the rotation speed of the cross-flow fan was $2000 \mathrm{r} / \mathrm{min}$, the blowing speed of the DJBW was $50 \mathrm{~m} / \mathrm{s}$, and the inflow velocity was $12 \mathrm{~m} / \mathrm{s}$, from which we can find that the lift drops sharply when $\alpha$ ranges from $20^{\circ}$ to $30^{\circ}$. With reference to the velocity contour of the blowing wing when its angle of attack was $30^{\circ}$ in Figure 16(b), it illustrates that the low-pressure vortex could not be formed in the arc groove of the DJBW and the airflow separation produced on the trailing edge of the wing causes the loss of lift. The loss of lift of the Fan-wing at a high angle of attack was less than that of the DJBW. With reference to the velocity contour of the Fan-wing when its angle of attack was $30^{\circ}$ in Figure 16(a), its low-pressure vortex was still kept near to the blade inside the cross-flow fan with a small size and the cross-flow fan does not have a good effect on the accelerating airflow from the slope section on the wing's upper surface. However, its lift decline is more gentle than that of the DJBW though the general trend was consistent with the blowing wing's where increase first and decrease later. 


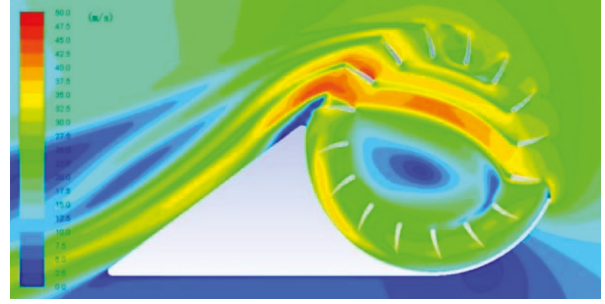

(a) Velocity contour of the Fan-wing

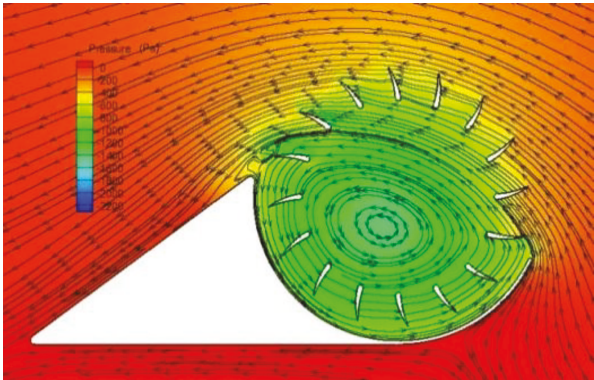

(c) Pressure contour and velocity flow chart of the Fan-wing

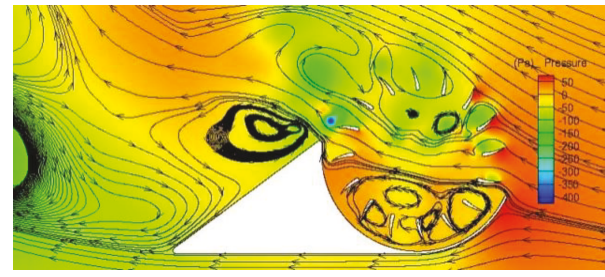

(e) Flow field of the cross-flow fan with a rotating speed of $0 \mathrm{~m} / \mathrm{s}$

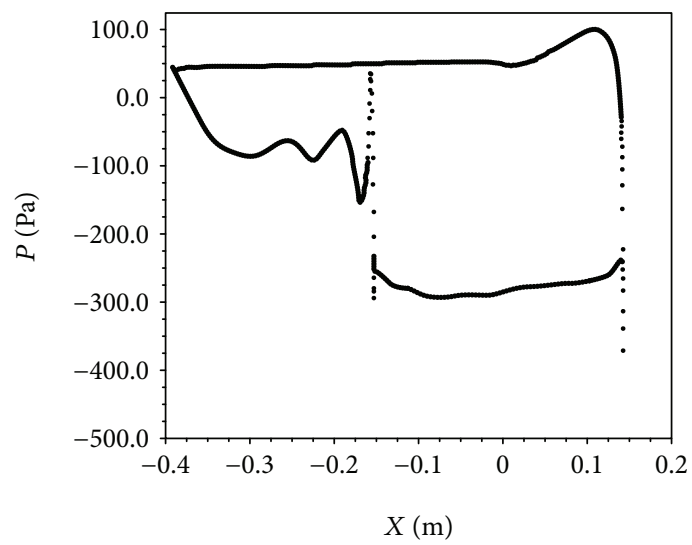

(g) Static pressure distribution curve of the Fan-wing

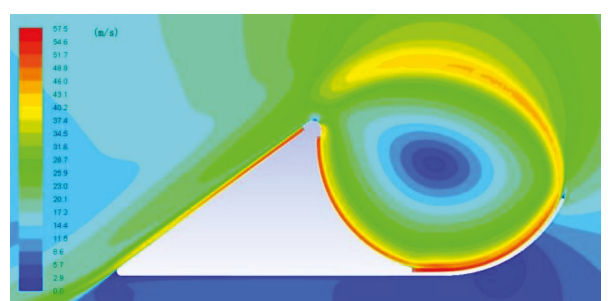

(b) Velocity contour of the DJBW

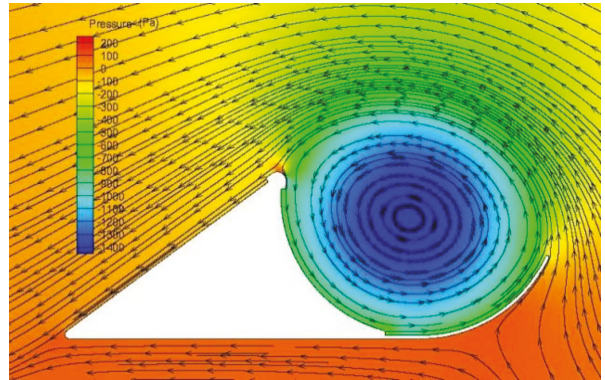

(d) Pressure contour and velocity flow chart of the DJBW

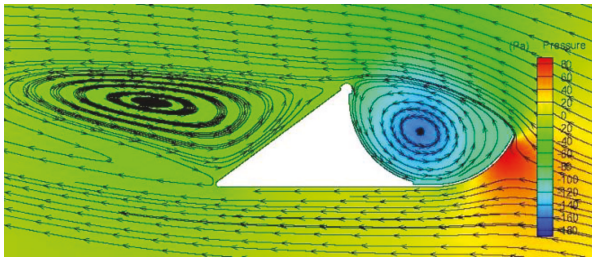

(f) Flow field of the blowing ports with a blowing velocity of $0 \mathrm{~m} / \mathrm{s}$

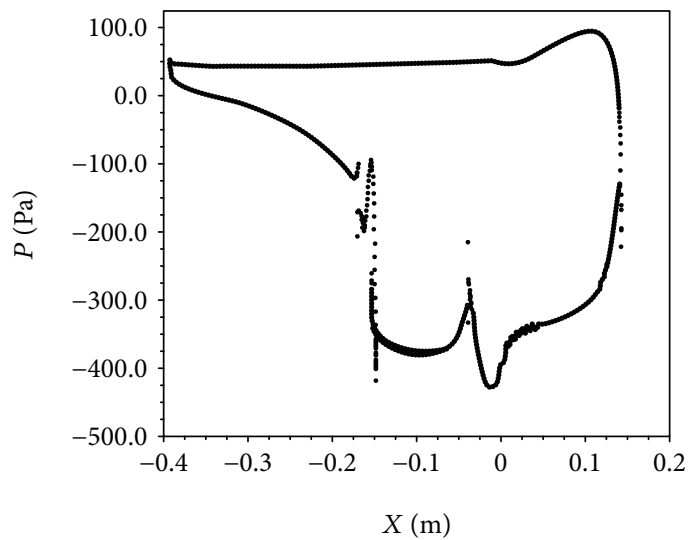

(h) Static pressure distribution curve of the DJBW

FIgURE 12: Comparisons of numerical calculation for two wings.

Figure 17 presents the relation curve showing how the thrust varies with the changing angle of attack $(\alpha)$ when the rotation speed of the cross-flow fan was $2000 \mathrm{r} / \mathrm{min}$, the blowing speed of the DJBW is $50 \mathrm{~m} / \mathrm{s}$, and the inflow velocity is $12 \mathrm{~m} / \mathrm{s}$, from which we can find that the thrust drops sharply with resistance produced when $\alpha$ ranges from $20^{\circ}$ to $30^{\circ}$. Although there was counterforce from blowing port 1 , it has a weak effect on producing thrust, which indicates that the low-pressure vortex in the arc groove of blowing wing contributes a lot to thrust. The Fan-wing loses less thrust at a huge angle of attack because on one hand there is counterforce as it is rotating and on the other hand there exists the low-pressure vortex in the arc groove.

Figure 18 presents the relation curve showing how the lift varies with the changing inflow velocity when the rotation speed of the cross-flow fan was $2000 \mathrm{r} / \mathrm{min}$, the blowing speed of the DJBW was $50 \mathrm{~m} / \mathrm{s}$, and the angle of attack was $20^{\circ}$, from which we can find that the lift of both the DJBW and Fan-wing grows as the inflow velocity increases and their variation trends are consistent with each other.

Figure 19 presents the relation curve showing how the thrust varies with the changing inflow velocity when the 


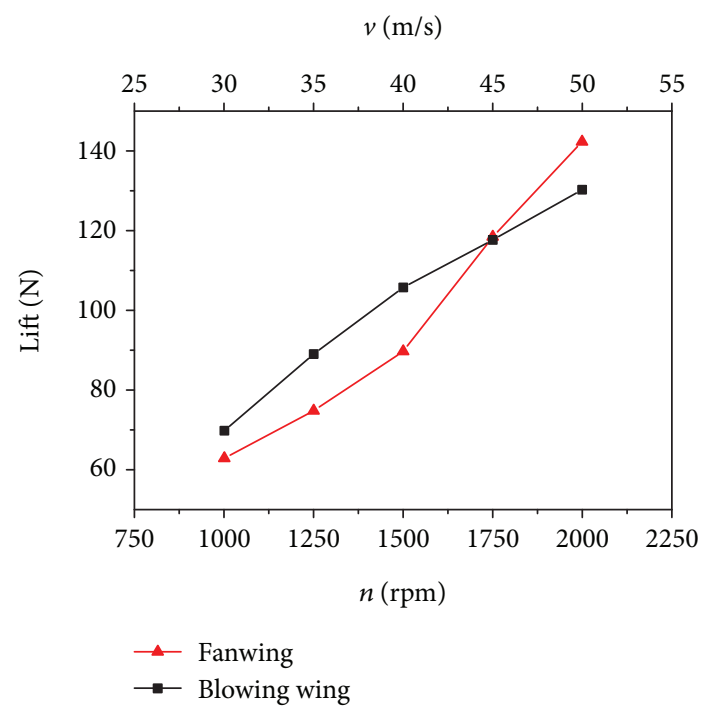

FIGURE 13: Lift curve changes with the rotating speed and blowing velocity.

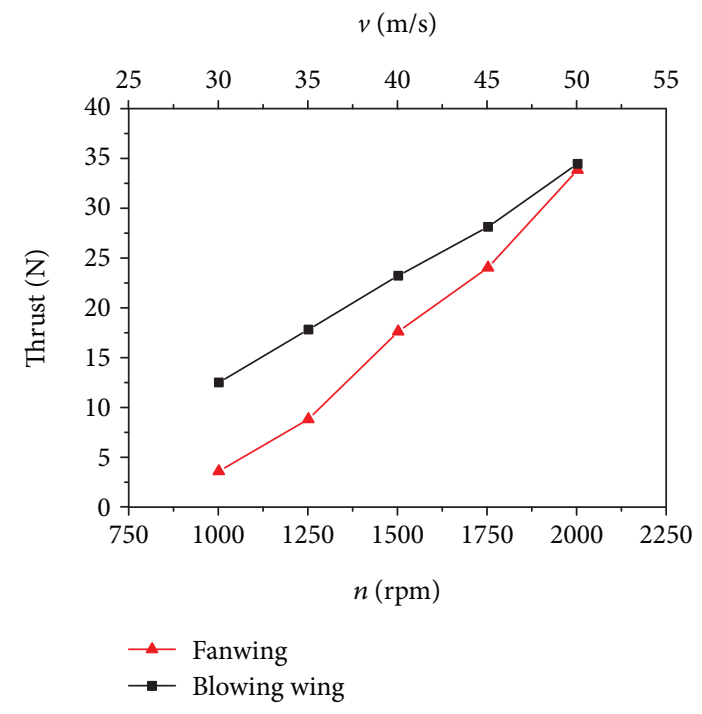

FIGURE 14: Thrust curve changes with the rotating speed and blowing velocity.

rotation speed of the cross-flow fan was $2000 \mathrm{r} / \mathrm{min}$, the blowing speed of the DJBW was $50 \mathrm{~m} / \mathrm{s}$, and the angle of attack was $20^{\circ}$, from which we can find that the thrust of the DJBW first increases and then decreases and the variation was not large for lift, and so was the Fan-wing. Therefore, the inflow velocity does not have a great effect on the thrust of the two kinds of wing.

\section{Conclusion and Discussion}

Based on the analysis of the principle of the lift and thrust of the Fan-wing, a DJBW was introduced in this study. By the method of numerical simulation, the aerodynamic

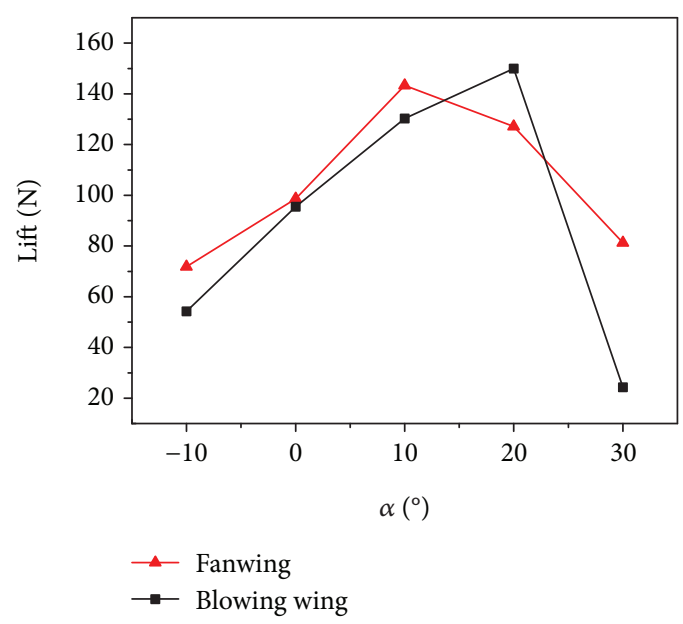

FIGURE 15: Lift curve changes with the angle of attack.

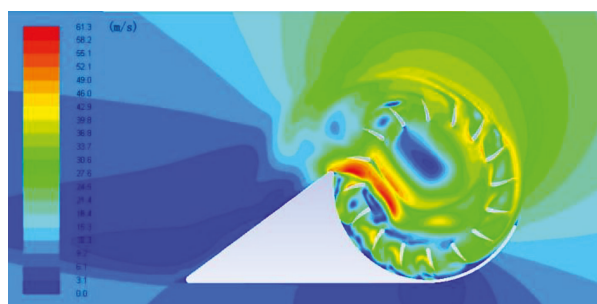

(a) Fan-wing

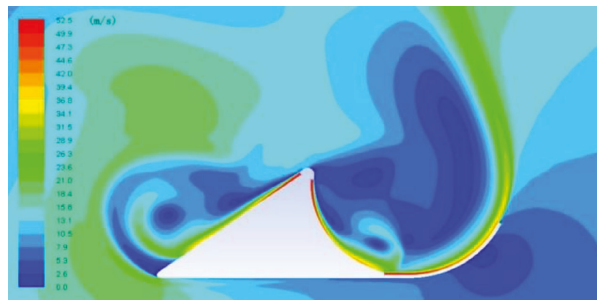

(b) DJBW

FIgURE 16: Velocity contour of two wings.

characteristics of the jet blowing wing and the Fan-wing were compared and analyzed, and the following conclusions are obtained:

(1) On producing the lift, the low-pressure vortex can be formed inside the arc groove of the blowing wing, from which the vortex lift was the main source of the wing's lift. This form of lift was consistent with that of the Fan-wing. In some cases, the aerodynamic effect of the blowing wing was more advantageous than that of the Fan-wing

(2) On producing the thrust, the high angle of attack has a great effect on the thrust of the blowing wing and the low-pressure vortex inside the arc groove was the main source of the thrust of the blowing wing. In the range of conventional angle of attack, the thrust effect of the blowing wing was similar to that of the Fan-wing 


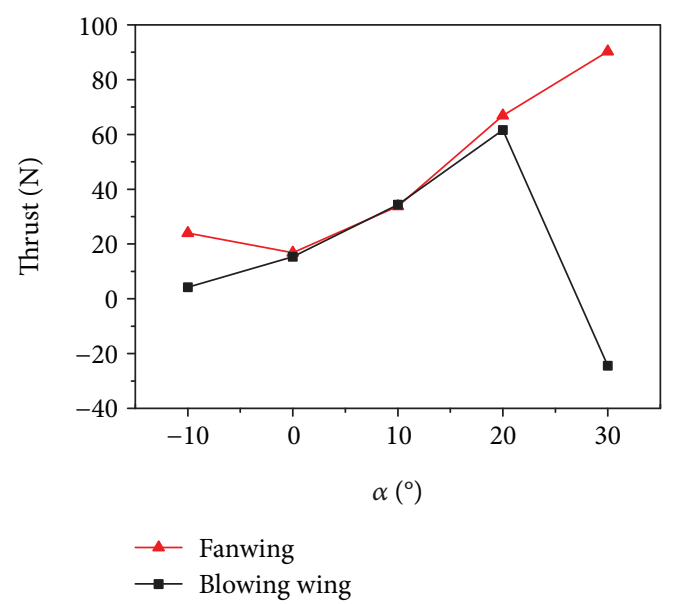

Figure 17: Thrust curve changes with the angle of attack.

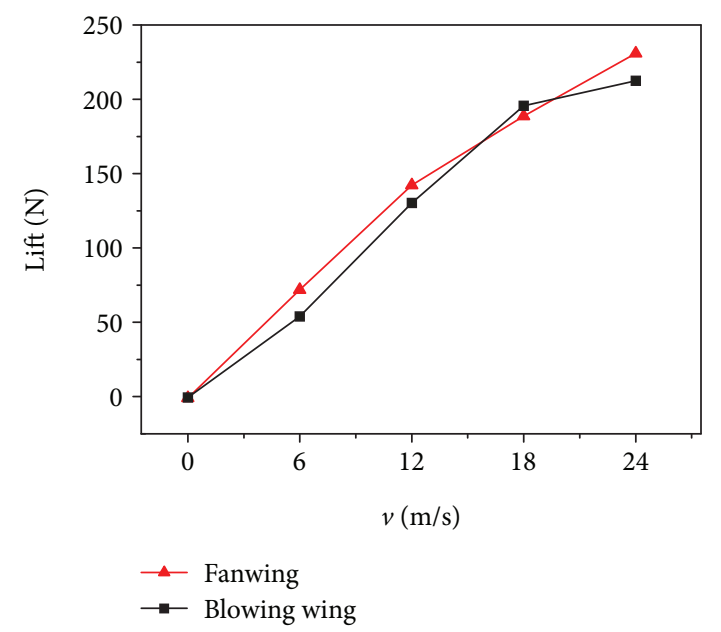

FIGURE 18: Lift curve changes with the forward flight speed.

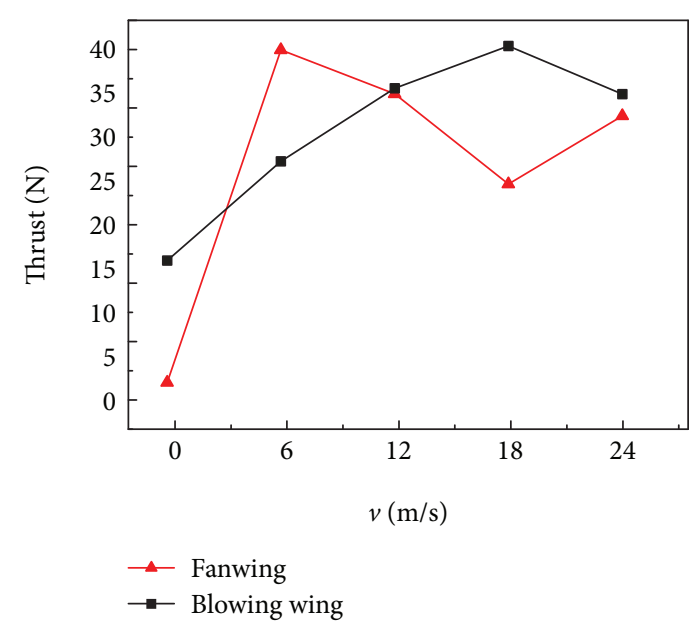

Figure 19: Thrust curve changes with the forward flight speed.
(3) The preliminary analysis shows that the DJBW can form aerodynamic characteristics similar to that of the fan and has potential advantages in replacing the fan. Further analysis and comparison are needed

\section{Data Availability}

The data used to support the findings of this study are available from the corresponding author upon request.

\section{Conflicts of Interest}

The authors declare that there is no conflict of interest regarding the publication of this paper.

\section{Acknowledgments}

This project is supported by the Fundamental Research Funds for the Central Universities (Grant No. NS2016014), the China Postdoctoral Science Foundation (Grant No. 2018M642241), and the National Key Laboratory of Rotorcraft Aeromechanics.

\section{References}

[1] J. I. Hileman, Z. S. Spakovszky, M. Drela, M. A. Sargeant, and A. Jones, "Airframe design for silent fuel-efficient aircraft," Journal of Aircraft, vol. 47, no. 3, pp. 956-969, 2010.

[2] F. Kirsten, "Cycloidal propulsion applied to aircraft," Transactions of the American society of Mechanical Engineers, vol. 50, no. AER-50-12, pp. 25-47, 1928.

[3] J. B. Wheatley and R. Windler, "Wind tunnel tests of a cyclogiro rotor," NACA Technical Note, vol. 528, pp. 1-29, 1935.

[4] M. Benedict, M. Ramasamy, and I. Chopra, "Improving the aerodynamic performance of micro-air-vehicle-scale cycloidal rotor: an experimental approach," Journal of Aircraft, vol. 47, no. 4, pp. 1117-1125, 2010.

[5] H. Yu, L. K. Bin, and T. W. Beng, "The investigation of cyclogyro design and the performance," in Proceedings of International Council of the Aeronautical Sciences Meeting, ICAS 2006-1.3.3, Hamburg, 2006.

[6] G. R. Seyfang, "Fanwing-developments and applica-tions," in 28th Congress of International Council of the Aeronautical Sciences, pp. 1-9, ICAS, Brisbane, 2012.

[7] H. S. Bayindir and P. Guillermo, "Analysis of the flow field around the wing section of a FanWing aircraft under various flow conditions," in 53rd AIAA Aerospace Sciences Meeting, p. 1936, AIAA, Florida, 2015.

[8] G. R. Seyfang, "Recent developments of the Fan-wing aircraft," in The International Conference of the European Aerospace Socoeties, pp. 1-7, CEAS, Venice, 2011.

[9] P. Peebles, "Aerodynamic lift generating device," 2003, US Patent 527229.

[10] S. Foreshaw, Wind Tunnel Investigation of the New Fan-Wing Design, Imperial College, London, 1999.

[11] K. U. Kogler, "FANWING-experimental evaluation of an ovel lift \& propulsion device. MEng.thesis," Aeronautical Engineering Department, Imperial College, London, 2002.

[12] D. Siliang, T. Zhengfei, X. Pei, and J. Mengjiang, "Study on helicopter antitorque device based on cross-flow fan 
technology," International Journal of Aerospace Engineering, vol. 2016, Article ID 5396876, 12 pages, 2016.

[13] D. Siliang and T. Zhengfei, "The aerodynamic behavioral study of tandem fan wing configuration," International Journal of Aerospace Engineering, vol. 2018, Article ID 1594570, 14 pages, 2018.

[14] S. L. Du, Z. M. Lu, and Z. F. Tang, "Numerical simulation research on the boundary control method of the fanwing' $s$ airfoil," Acta aeronautica et Astronautica Sinica, vol. 37, no. 6, pp. 1783-1791, 2016.

[15] S. L. Du, R. P. Tang, and Z. F. Tang, "Experimental study on aerodynamic characteristics of fanwing [J]," Journal of Nanjing University of Aeronautics \& Astronautics, vol. 49, no. 3, pp. 403-410, 2017.

[16] L. Prandtl, "Early developments of modern aerodynamics," in English Translation, J. A. K. Ackroyd, B. P. Axcell, and A. I. Ruban, Eds., p. 77, Butterworth-Heinemann, Oxford, UK, 2001.

[17] H. Schlichting and K. Gersten, Boundary-Layer Theory, Springer, 8th edition, 2003.

[18] A. M. O. Smith, "High-lift aerodynamics," Journal of Aircraft, vol. 12, no. 6, pp. 501-530, 1975.

[19] V. CIOBACA and J. WILD, "An overview of recent DLR contributions on active flow-separation control studies for high-lift configurations," Aerospace Lab Journal, vol. AL0612,2013

[20] M. X. Wang, W. P. Sun, and H. J. Qin, "Optimization design of an internal blown flap used in large amphibian[J]," Acta aeronautica et Astronautica Sinica, vol. 37, no. 1, pp. 300-309, 2016. 


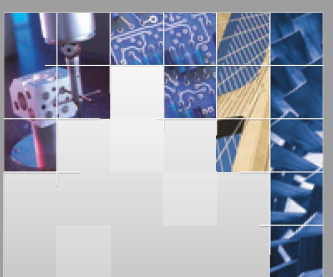

\section{Enfincering}
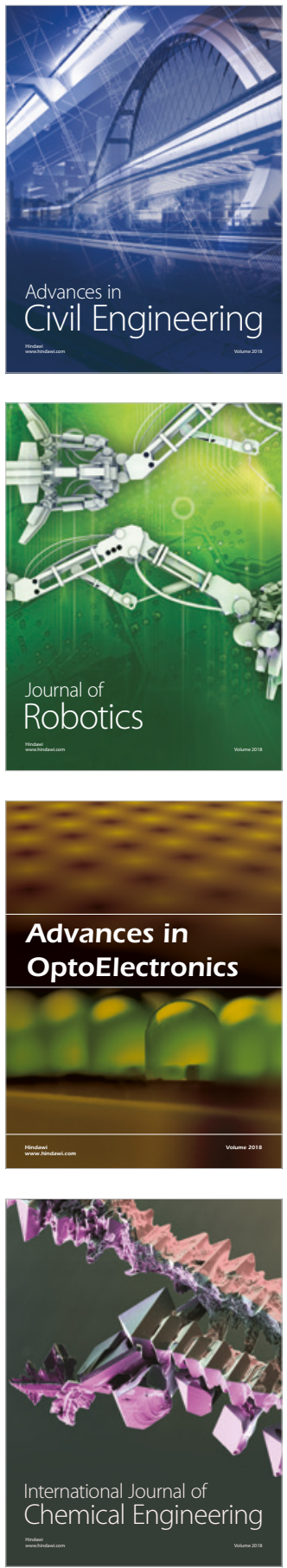

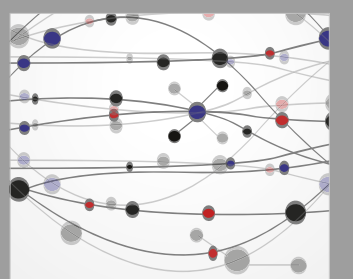

\section{Rotating \\ Machinery}

The Scientific World Journal

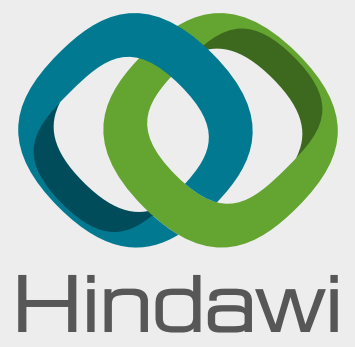

Submit your manuscripts at

www.hindawi.com
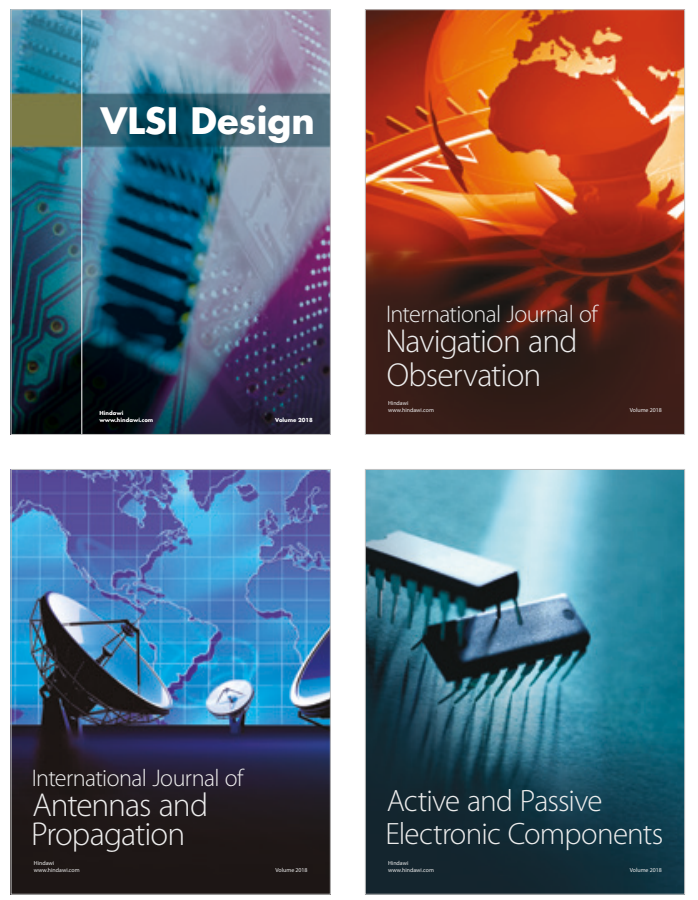
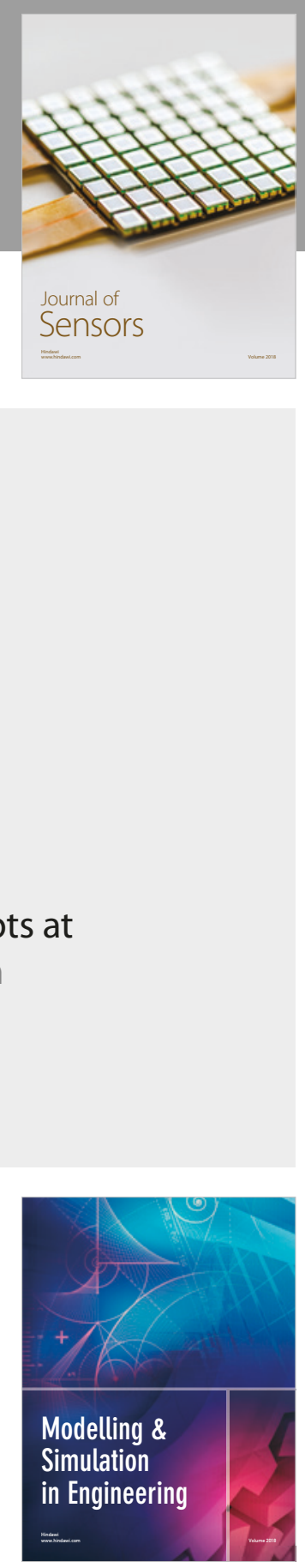

\section{Advances \\ Multimedia}
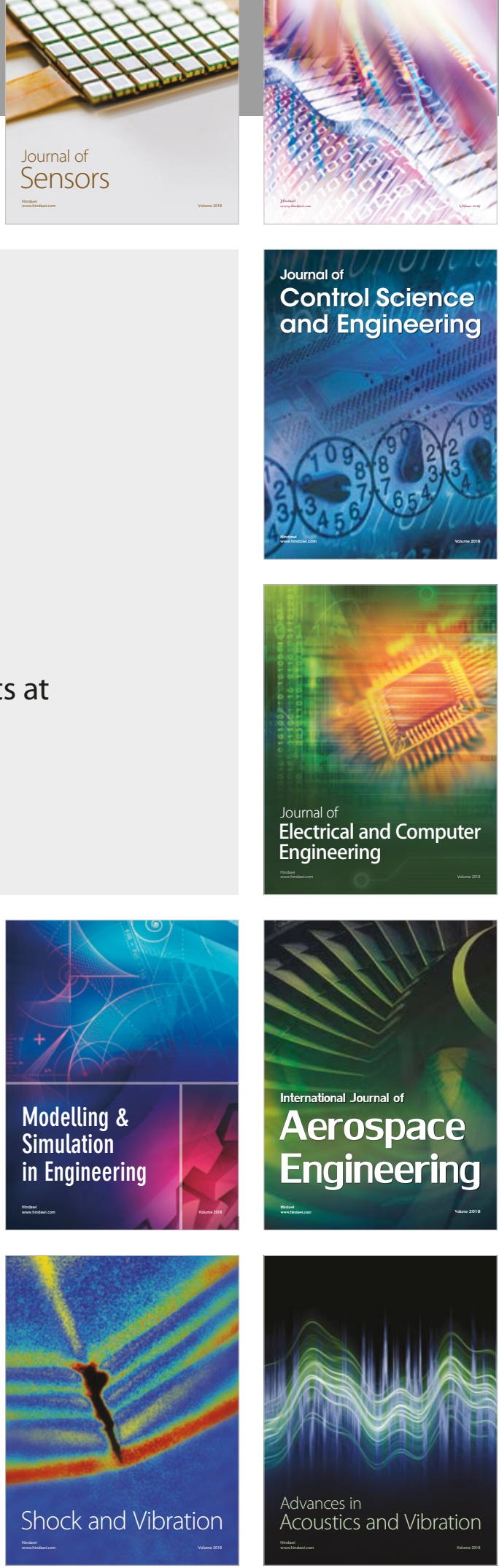